\title{
A New Age of Ocean Exploration
}

IN THE WORDS of Paul Simon, "these are the days of miracles and wonder." ${ }^{1}$ We live in a time when any given cruise can give rise to whole new concepts and observations that enhance understanding. Many of us recall the news that spread like wildfire from the 1970s expeditions to the Galápagos Rift. Geologists brought back huge tubeworms and other previously unseen organisms they found thriving in mid-ocean ridge hydrothermal vent fields. More recently, some of our colleagues retrieved in situ photographs of the 2-m squid, a species of Mastigoteuthis, previously undescribed. Images of this organism in its habitat had never been obtained before. Imagine the media fest that would ensue should such a discovery be made on land!

All of this simply emphasizes the extraordinary and fantastic world that is our field site. This issue of Oceanography gives us a taste of the ongoing magnificence and diversity of discovery in the field of ocean exploration. New species, new habitats, new processes, from descriptions of discoveries of deep corals to photographic evidence for the emission of liquid carbon dioxide from the seafloor ... all are part of the portfolio of exploration. The common thinking that exploration is limited to the spatial domain is refuted by the breadth of material covered in these pages. Exploration means the discovery of much more than just places, critters, and bugs!

And what about the technology? This too is a treasure trove of new platforms, sensors, and operational concepts. Real-time video feeds via satellite, then over Internet 2 from the deepest and most remote parts of the seafloor? Infrared cameras that work like spy cams on the secret world of marine organisms? Intelligent autonomous vehicles with technology rivaling that of the most sophisticated planetary exploration vehicles? If these capabilities don't excite the next generation of ocean scientists, I don't know what will. I'm also convinced we could have quadrupled the size of this issue of Oceanography and still not have captured all of the exciting new technologies that form a foundation for ocean exploration.
But even with all of this "buzz," ocean exploration still has an identity crisis. Is it science? Is it research? How do we balance what is - to some critics—an unfettered stroll about the world's seas looking for new things with a need to focus our limited resources on those highest priorities for hypothesis-based application of the scientific method? It's a tough question, and one that I believe is founded in our willingness to take some risks in how we spend our resources. Sure, we shouldn't just put the creative scientists aboard a research vessel, give them all the toys they ask for, cast the lines, and wish them well in their search for exciting discoveries. By the same token, we shouldn't hold these same courageous explorers hostage to forecasting exactly what they will find in order to justify every hour of cruise time they are afforded. There is a happy medium. I believe that medium is defined in terms of a balance of expectations and tolerance for some serendipity.

Three decades ago, when the team of marine geologists went to the Galápagos Rift, they had good reason to believe they would find new phenomena on the ocean floor. The investment in their exploration was based on a good set of scientific questions. Their discovery of a realm of organisms and biological processes so unexpected was made the more glorious by its serendipity (imagine how even more glorious it might have been had they had the foresight to invite some biologists aboard!). The point is that we should be structured in ocean exploration to both drive the research agenda, and be driven by it. The papers in this issue of Oceanography demonstrate that beautifully.

As one who has watched the evolution of ocean exploration, I am excited by what the next several decades will bring. With NOAA's new dedicated ocean exploration vessel, Okeanos Explorer, coming on line in the fleet within a year, and the formal establishment of an ocean exploration program, I am convinced that Paul Simon's words will continue to ring true for quite some time.

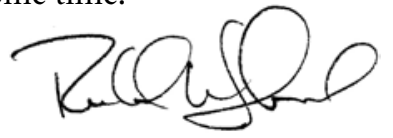

RICHARD SPINRAD, TOS PRESIDENT

"The Boy in the Bubble" @ 1986 Paul Simon Music by Paul Simon and Forere Motloheloa. 